\title{
Obstetric Anal Sphincter Injuries - Maternal, Foetal and Sociodemographic Risk Factors: A Retrospective Register-Based Study
}

\author{
Kristin André ${ }^{1}$, Andrea Stuart ${ }^{2}$, and Kärin Kallén ${ }^{3}$ \\ ${ }^{1}$ Affiliation not available \\ ${ }^{2}$ Helsingborgs lasarett \\ ${ }^{3}$ Lund University Clinical Sciences
}

October 19, 2021

\begin{abstract}
Objective. To determine risk and protective factors of obstetric anal sphincter injuries (OASIS). Design. A retrospective register-based observational study. Setting. Sweden. Population. A cohort of 988, 988 singleton term deliveries 2005-2016 were included. Methods. Data from the Swedish Medical Birth Registry and Statistics Sweden were extracted to identify cases of OASIS and maternal and foetal characteristics. Modified Poisson Regression analyses were performed to assess risk factors. Main outcome measures. Risk ratios for OASIS with 95\% confidence interval associated with maternal and foetal risk factors were calculated. Results. The rate of OASIS was 3.5\% ( $\mathrm{n}=34,583$ ). Primiparity (aRR 3.13 95\% CI 3.05-3.21), vacuum extraction (aRR 2.79 95\% CI 2.73-2.86), forceps (aRR 4.27 95\% CI 3.86-4.72) and high birth weight (aRR 2.61 95\% CI 2.50-2.72) were associated with a significantly increased risk of OASIS. Increasing maternal age and decreasing maternal height increased the risk of OASIS. Smoking (aRR $0.7495 \%$ CI 0.70-0.79) and low maternal education (aRR $0.8795 \%$ CI 0.83-0.92) were associated with a decreased frequency of reported OASIS. Obesity decreased the risk of OASIS (aRR $0.9095 \%$ CI $0.87-0.94$ ), but only after adjusting for foetal birth weight. Previous caesarean section increased the risk of OASIS (aRR 1.41; 95\% CI 1.36-1.47). Conclusion. Primiparity, instrumental delivery and high birth weight increased the risk of OASIS. Risk factors including BMI, height, age, smoking, maternal education, ethnicity and previous caesarean section also contribute to the overall risk of OASIS. Keywords. Obstetric sphincter injuries, risk factors, pregnancy.
\end{abstract}

\section{Obstetric Anal Sphincter Injuries - Maternal, Foetal and Sociodemographic Risk Factors: A Retrospective Register-Based Study}

\section{Kristin André}

Department of Obstetrics and Gynaecology, Helsingborg Central Hospital, Helsingborg, Sweden

\section{Andrea Stuart}

Department of Obstetrics and Gynaecology, Helsingborg Central Hospital, Helsingborg, Sweden Institution of Clinical Sciences Lund, Lund University, Lund, Sweden

\section{Karin Källén}

Institution of Clinical Sciences Lund, Lund University, Lund, Sweden

Centre for Reproductive Epidemiology, Tornblad Institute, Lund University, Lund, Sweden

\section{Corresponding author:}


Kristin André

Department of Obstetrics and Gyneacology, Helsingborgs Lasarett.

Charlotte Yhléns gata 10, 25223 Helsingborg, Sweden

$+46734233405$

kristin.c.andre@gmail.com

Running title: Risk Factors for Obstetric Anal Sphincter Injuries

Abstract

Objective . To determine risk and protective factors of obstetric anal sphincter injuries (OASIS). Design . A retrospective register-based observational study. Setting . Sweden. Population . A cohort of 988, 988 singleton term deliveries 2005-2016 were included. Methods . Data from the Swedish Medical Birth Registry and Statistics Sweden were extracted to identify cases of OASIS and maternal and foetal characteristics. Modified Poisson Regression analyses were performed to assess risk factors. Main outcome measures . Risk ratios for OASIS with 95\% confidence interval associated with maternal and foetal risk factors were calculated. Results . The rate of OASIS was 3.5\% ( $\mathrm{n}=34,583)$. Primiparity (aRR $3.1395 \%$ CI 3.05-3.21), vacuum extraction (aRR 2.79 95\% CI 2.73-2.86), forceps (aRR 4.27 95\% CI 3.86-4.72) and high birth weight (aRR 2.61 95\% CI 2.50-2.72) were associated with a significantly increased risk of OASIS. Increasing maternal age and decreasing maternal height increased the risk of OASIS. Smoking (aRR 0.74 95\% CI 0.70-0.79) and low maternal education (aRR $0.8795 \%$ CI 0.83-0.92) were associated with a decreased frequency of reported OASIS. Obesity decreased the risk of OASIS (aRR $0.9095 \%$ CI 0.87-0.94), but only after adjusting for foetal birth weight. Previous caesarean section increased the risk of OASIS (aRR 1.41;95\% CI 1.36-1.47). Conclusion . Primiparity, instrumental delivery and high birth weight increased the risk of OASIS. Risk factors including BMI, height, age, smoking, maternal education, ethnicity and previous caesarean section also contribute to the overall risk of OASIS.Keywords . Obstetric sphincter injuries, risk factors, pregnancy.

\section{Tweetable abstract}

Primiparity, instrumental delivery and VBAC increased the risk of OASIS, whilst maternal smoking and low educational level was associated with lower incidences of reported OASIS.

\section{Introduction}

Obstetric anal sphincter injuries (OASIS) are serious complications to vaginal deliveries. They represent the most significant risk factor for developing female anal incontinence and might also cause perineal pain, dyspareunia, faecal urgency and urinary incontinence, leading to significant long-term suffering and potential stigmatisation.

Perineal injuries are classified according to a definition published by Sultan. First and second-degree injuries involve the vaginal epithelium and perineal muscles respectively. OASIS include third- and fourth-degree injuries to the perineum involving the external anal sphincter (EAS), internal anal sphincter (IAS), and the anal epithelium to varying extent.

There are several well-established risk factors associated with a higher risk of OASIS, including primiparity, foetal macrosomia, instrumental vaginal delivery, and prolonged second stage of delivery. Other aspects that have been proposed to affect the risk include maternal age, height, weight, smoking, ethnicity and educational level, but the evidence has either been sparse or not consistent in published material.

The prevalence of sphincter injury has fluctuated over the last decades and varies across countries. The incidence varies between 0.7-4.2\% in the Nordic countries, with Finland representing the lowest and Denmark the highest numbers. An increasing trend has been observed where several explanations have been proposed; larger infant size, increased maternal age, increase in instrumental deliveries, improved diagnostic accuracy, and changed clinical practice of management of second stage of delivery. 
Despite OASIS being recognised as a significant cause of both long-, and short-term morbidity there is no international consensus regarding the use of preventative measures. Several factors modifiable during labour have been investigated. Controlled delivery of the head, warm compresses held against the perineum, and choice of delivery position all have shown to decrease the risk of OASIS. The evidence regarding perineal protection manoeuvres has not been proven to have an effect in clinical studies but is used on a routine basis in clinical practice in Sweden and has been part of interventional programs that have successfully reduced the incidence of OASIS. For women at very high risk of OASIS a planned caesarean section might be offered as a preferred route of delivery.

Identifying risk factors in order to provide optimal preventative measures for high-risk women could lead to a significant decrease in long-term suffering and stigmatised symptoms. The aim of this nationwide registerbased study was therefore to assess maternal, foetal and socio-demographic risk and protective factors for OASIS across the Swedish population.

\section{Methods and materials}

The study population consisted of 988,988 women with singleton term ([?]37+0) vaginal deliveries in Sweden 2005-2016. The data was extracted from the Swedish Medical Birth Registry (MBR), which was founded in 1973 and includes data on almost all deliveries (98-99\%) in Sweden. All health care providers are obliged to report to the registry and the system is identical throughout the country. It contains information collected from medical records registered at antenatal visits, delivery units and initial paediatric examination of the infant. A detailed quality control and validation of the registry was published in 2003.

At the first antenatal visit the women are interviewed by their midwife regarding their pre-pregnancy health, including smoking habits and use of medical drugs. Also, the height and their booking weight are registered. Maternal Body Mass Index (BMI) was calculated through weight $(\mathrm{kg}) /(\text { height }(\mathrm{m}))^{2}$, and was classified according to WHO's classification into underweight (BMI $<18.5$ ), normal weight (BMI 18.5 - 24.9), overweight (BMI 25-29.9), and obesity (BMI [?] 30). Information regarding maternal educational level and maternal country of birth was obtained through linkage with data from Statistics Sweden. Cases of OASIS were identified through checkboxes, or ICD-code O702 and O703 reported to the MBR or the National Patient Registry (NPR) within six weeks after delivery.

\section{Statistics}

Risk Ratios (RR) and Adjusted Risk Ratios (aRR) for sphincter rupture were obtained using modified Poisson Regression analyses (SPSS version 25). When specified, adjustments were made for year of delivery (classes 2005-2008, 2009-2012, 2013-2016) and maternal characteristics: Maternal age (classes $<20,20-$ $24.9,25-29.9,30-34.9,35-39.9$, or [?]40 years), parity (1, 2, or [?]3 children), BMI classes (<18.5, 18.5-24.9, 25-29.9, [?]30, or not known), smoking (yes, no, or not known), maternal height classes $(<155,155-164$, 165-174, [?]175 cm, or not known), maternal country of birth (Nordic countries, other European countries/USA/Canada/Australia/New Zeeland, others, or not known), and maternal educational level ([?]primary/lower secondary (1-9 years), upper secondary (10-12 years), [?]3 years higher education, [?]3 years higher education, or not known). When specified, adjustments were also made for delivery mode (vaginal non-instrumental, vacuum extraction, or forceps), and/or infant birth weight $(<2500,2500-2999,3000-3499$, 3500-3999, 4000-4499, [?]4500g). Findings with p-values $<0.05$ were regarded as statistically significant.

\section{Results}

Between 2005-2016, 988988 vaginal deliveries were recorded in the MBR. The rate of OASIS was 3.5\% $(\mathrm{n}=34$ 583). Table 1 shows maternal characteristics and delivery mode in relation to the risk of OASIS. There was an overall seven percent decrease of OASIS during 2013-2016 as compared to 2005-2008.

The risk of OASIS increased with maternal age as seen after adjusting for maternal characteristics (Table 2 ), with the highest risk in the age group 34-39 years, and inversely young maternal age of $<20$ years was significantly protective. When also adjusting for delivery mode, the association between OASIS and increasing maternal age was less pronounced, albeit still statistically significant. 
Primiparous women were approximately three times as likely to attain OASIS as compared to multiparous women, and the strong association between primiparity and OASIS remained after adjustments.

In the crude model, high BMI (>30) had a considerably protective effect (cRR 0.80), but after initial adjustment for maternal characteristics and then delivery mode this association disappeared. After also adjusting for birth weight a negative association was again seen, but less pronounced (aRR 0.90). Low BMI $(<18.5)$ showed a risk increase of OASIS as compared to women with normal BMI (cRR 1.12). Through all three steps of adjustment the risk increase among women with BMI $<18.5$ was maintained, and the positive association was even more noticeable (aRR 1.30). Maternal height showed an inverted relationship to risk of OASIS which became more evident after adjusting both for maternal characteristics, delivery mode and birth weight (height $<155 \mathrm{~cm}$ : aRR 1.72 , compared to height $>175 \mathrm{~cm}$ : aRR 0.82 ).

Maternal smoking appeared to be negatively associated with risk of OASIS that remained after adjusted calculations, even when adjusting for birth weight (aRR 0.74). An association between increased risk of OASIS and higher maternal educational level was found, and adjustment for maternal characteristics, delivery mode, and birth weight only marginally altered the risk estimates. According to the crude estimates, no association between maternal country of birth and OASIS was found, but when adjustments were made, decreased risk (aRR 0.92) was found for women from Europe/ USA/ Canada/ Australia/ New Zeeland as compared to women from the Nordic countries, whereas women with other ethnic origin had a significant risk increase (aRR 1.31) as compared to women from the Nordic countries.

The strongest risk factor for OASIS was instrumental delivery, where the most significant risk increase was seen with the use of forceps (cRR 6.88). The prevalence of vacuum extraction (VE) was $8.3 \%$ and the use of forceps was rare at $0.2 \%$.

Vaginal birth after previous caesarean section (VBAC) as a risk factor of OASIS was analysed separately and the risk of OASIS among secundiparous women with a vaginal birth following one previous caesarean section $(2,755 / 29,927)$ was compared to the corresponding risk among primiparous women with vaginal births $(25,576 / 429,727)$. This analysis showed a profound risk increase (cRR 1.60; 95\% CI 1.54-1.66) of OASIS after previous CS. After adjustment for maternal characteristics the risk of OASIS remained significantly elevated (aRR 1.41; 95\% CI 1.36-1.47). This risk increase could partly be explained by an increase in instrumental delivery, but after adjustment for delivery mode and birth weight the risk remained raised (aRR 1.24; $95 \%$ CI 1.20-1.29).

\section{Discussion}

Our results support the increased risk of OASIS related to well-established risk factors, including primiparity, instrumental delivery and high birth weight. We showed an association with increasing maternal age, obesity (if unadjusted for birth weight), lower maternal height and previous CS. Smoking and low maternal education level were negatively associated with OASIS.

Increasing BMI showed a decreased risk of OASIS in the crude analysis, however only apparent in the adjusted model after adding birth weight to the analysis. Obese women are more likely to give birth to larger babies and it is therefore important to consider whether this apparent protective effect is clinically relevant, or if their overall risk of OASIS is instead increased secondary to risk of foetal macrosomia as seen in the unadjusted results. A decreased risk of OASIS was seen in two previous Swedish studies, with a more profound risk reduction in BMI $>35$. Constable et al., showed a risk reduction (aOR 0.27) among women with BMI $>35$ in an Australian setting, and Garetto et al. observed an aOR 0.75 in obese American women. All previously mentioned studies either adjusted for birth weight or excluded cases with babies $>4000 \mathrm{~g}$. On the contrary, other studies have not been able to statistically detect any protective effect, whereas Landy et al., only found a risk decrease among nulliparous women.

The proposed underlying mechanisms of a protective effect include an increased amount of adipose tissue making the perineal tissue softer and more stretchable, and a larger perineal body increasing the anovaginal distance. These hypotheses are still to be scientifically proven. In contrast, perineal tissue has been suggested 
to be weaker in obese women; higher BMI has been associated with striae gravidarum which in turn has been linked to a higher risk of perineal trauma. Another consideration is potentially increased missed diagnoses relating to difficulties in examining women with voluminous amount of adipose tissue.

Women with $<9$ years education had a $15 \%$ lower risk of reported OASIS as compared to those with $>3$ years of higher education after adjusting for maternal characteristics. Similar results are seen in a Finnish study where higher socioeconomic status (SES) was associated with an $18 \%$ increased risk of OASIS. A British study showed that women of high SES were more than twice as likely to suffer from OASIS as compared to those of low SES. This difference is somewhat surprising and difficult to explain. It either stands for an unknown confounding factor not accounted for, or potentially by a difference in the provision of health care. The higher risk in women with higher educational level could instead represent a lower risk of missed diagnosis.

We found smoking to be negatively associated with OASIS (cRR 0.49), remaining after adjusting for maternal characteristics, and surprisingly also after adjusting for delivery mode and birth weight (aRR of 0.74). Similar results are seen in a Finnish study where smoking decreased the incidence of OASIS in primiparous women by $28 \%$, and by $20 \%$ in a previous Swedish study among primiparous women. The underlying mechanisms of the apparent protective effect are yet unexplained. The results appear paradoxical to other known effects of smoking such as premature skin ageing, reduced blood flow, and an accumulation of body-wide oxidative damage. This causes us to speculate around the true protective effect of smoking, or whether a difference in provision of health care can explain the results.

Maternal age was inversely related to risk of OASIS where women $<20$ years of age had a $50 \%$ lower risk of OASIS compared to women aged 24-29 years. Women $>35$ years on the other hand, had an approximately $20 \%$ risk increase. The highest age group $(>40)$ had a slightly lower risk increase after adjustments than women aged 34-39, but the difference between the risk estimates was far from significant $(\mathrm{p}=0.20)$. GurolUrgandi et al., showed a similar risk decrease in teenage mothers, but only a slight risk increase (aOR 1.07) in women aged 30-34. A previous Swedish register-based study showed the risk of OASIS to increase almost continuously with age irrespective of parity, with exception for nulliparous women where the risk did not further increase $>35$ years which was similar to our findings. In this previous study, the aOR was around 2 for nulliparous women $>30$ years, however the reference group differed from ours $(<25$ years) which could explain the difference in calculated risks. Similarly, Hornemann et al. found a higher maternal age in women with OASIS compared to women with less severe perineal lacerations. The specific effects of ageing on perineal tissue have been poorly researched and age-specific advantage of protective measures have not been studied. Considering the trends in modern society to start a family at an older age the risk should be acknowledged, and attention should be given to the importance of accurate diagnosis and treatment.

Maternal height was inversely related to risk of OASIS. Women $<155 \mathrm{~cm}$ had an aRR of 1.34 after adjusting for maternal characteristics. We stipulated adjustments for delivery mode and birth weight would lessen this association, but the correlation instead became more profound and aRR increased to 1.72, suggesting maternal height as a significant independent risk factor. Raisanen et al. found an increase of $1 \mathrm{~cm}$ in maternal height to decrease the risk of sphincter injury by $2 \%$. However, there is limited research on the effects of maternal height on obstetric outcomes.

Our results show an increased risk of OASIS among women achieving VBAC compared to primiparous women. D'Souza et al. found a similar risk of OASIS after VBAC compared to that of primiparous women, and a 6.8-fold risk increase in secundiparous women as compared to multiparous women with previous vaginal births only. Raisanen et al. found a 1.4-fold risk increase associated with VBAC and propose an underlying foetopelvic disproportion as a potential explanation, putting women at risk of initial CS and subsequently of OASIS in following vaginal deliveries.

Strengths of this study include using a population-based register providing a large study population available for inclusion. This enabled analysis of multiple risk factors to produce reliable results. The Swedish MBR is of high quality with $<2 \%$ missing data and is mandatory across the population providing non-selective 
standardised data. Nevertheless, the register contains some errors and missing values. We also recognise that the classification and diagnosis of OASIS is subjective and depends on the care provider and local routine. Furthermore, our analysis did not include foetal head circumference, length of second stage of labour, use of anaesthetic or episiotomy rates which might impact the overall risk.

\section{Conclusion}

In this large register-based study we observed an increased risk of OASIS associated with primiparity, instrumental delivery, increasing maternal age, previous CS and low maternal height. Obesity was observed to slightly increase the risk of OASIS if adjustments for birth weight were not made, which might be the most clinically appropriate adjustment model to use in this case. A negative association with OASIS was seen with smoking and low maternal educational levels, however there are uncertainties whether this association represents a true protective effect or a potential difference in the provision of health care. Future studies will be able to use these results in a clinical setting aimed at individual risk assessment improving women's health.

Acknowledgements : None

Disclosure of interest : None to declare

Contributions of authorship : KA, AS and KK contributed to the overall design of the study and the analysis and interpretation of the data. KA drafted the first manuscript. AS and KK then revised and critically appraised the draft before all authors approved of the final manuscript.

Details of ethics approval : The study was approved by the Regional Medical Ethics Committee, Lund, Sweden $(2018 / 538)$.

Funding : None to declare

\section{References}

Table 1. Maternal characteristics and delivery mode in relation to the risk of OASIS

\begin{tabular}{|c|c|c|c|c|c|c|}
\hline & & OASIS & OASIS & All vaginal births & Crude & Crude \\
\hline & & $\mathrm{n}$ & $(\%)$ & $\mathrm{n}$ & $\mathrm{RR}$ & $(95 \%$ \\
\hline \multirow[t]{3}{*}{ Year of delivery } & $2005-2008$ & 12226 & $(3.6)$ & 335805 & 1.0 & Refe \\
\hline & 2009-2012 & 12370 & $(3.5)$ & 357174 & 0.95 & 0.93 \\
\hline & 2013-2016 & 9987 & $(3.4)$ & 296009 & 0.93 & 0.90 \\
\hline \multirow[t]{6}{*}{ Maternal age (years) } & $<20$ & 349 & $(2.3)$ & 15423 & 0.59 & 0.53 \\
\hline & $20-24$ & 3959 & (3.0) & 133787 & 0.77 & 0.74 \\
\hline & $24-29$ & 11522 & $(3.8)$ & 300127 & 1.0 & Refe \\
\hline & $30-34$ & 13037 & (3.8) & 341750 & 0.99 & 0.97 \\
\hline & $34-39$ & 4919 & (3.0) & 165330 & 0.78 & 0.75 \\
\hline & {$[?] 40$} & 797 & (2.4) & 32571 & 0.64 & 0.59 \\
\hline \multirow[t]{3}{*}{ Parity } & 1 & 25576 & (6.0) & 429727 & 2.81 & 2.74 \\
\hline & 2 & 7871 & $(2.1)$ & 371933 & 1.0 & Refe \\
\hline & {$[?] 3$} & 1136 & $(0.6)$ & 187338 & 0.29 & 0.27 \\
\hline \multirow[t]{3}{*}{ Smoking } & Yes & 1077 & $(1.8)$ & 60340 & 0.49 & $0.47-0$ \\
\hline & No & 31891 & (3.6) & 882298 & 1.0 & Refe \\
\hline & Not known & 1615 & $(3.5)$ & 46360 & 0.96 & 0.92 \\
\hline \multirow[t]{5}{*}{ Body Mass Index $\left(\mathrm{kg} / \mathrm{m}^{2}\right)$} & $<18.5$ & 951 & $(4.0)$ & 23543 & 1.12 & 1.05 \\
\hline & $20-24.9$ & 20428 & $(3.6)$ & 563735 & 1.0 & Refe \\
\hline & $25-29.9$ & 7563 & $(3.4)$ & 222729 & 0.94 & $0.91-$ \\
\hline & {$[?] 30$} & 3022 & $(2.9)$ & 103740 & 0.80 & 0.77 \\
\hline & Not known & 2600 & $(3.5)$ & 74627 & 0.96 & $0.92-1$ \\
\hline
\end{tabular}




\begin{tabular}{|c|c|c|c|c|c|c|}
\hline & & OASIS & OASIS & All vaginal births & Crude & Crude \\
\hline \multirow[t]{5}{*}{ Maternal height $(\mathrm{cm})$} & $<155$ & 1159 & $(4.2)$ & 27892 & 1.21 & $1.14-1$ \\
\hline & $155-164$ & 11704 & $(3.6)$ & 325729 & 1.05 & $1.02-1$ \\
\hline & $165-174$ & 16745 & $(3.4)$ & 488258 & 1.0 & Refe \\
\hline & {$[?] 175 \mathrm{~cm}$} & 3332 & $(3.3)$ & 99815 & 0.97 & 0.94 \\
\hline & Not known & 1643 & $(3.5)$ & 47298 & 1.01 & $0.96-$ \\
\hline \multirow[t]{5}{*}{ Maternal educational level } & [?]Primary/lower secondary & 1972 & $(2.3)$ & 86794 & 0.55 & $0.52-0$ \\
\hline & Upper secondary & 9675 & $(2.9)$ & 333887 & 0.70 & 0.68 \\
\hline & $<3$ yrs higher education & 4280 & $(3.6)$ & 119622 & 0.86 & $0.83-$ \\
\hline & [?]3 yrs higher education & 15999 & $(4.2)$ & 385437 & 1.00 & Refe \\
\hline & Not known & 2657 & $(4.2)$ & 63258 & 1.01 & 0.97 \\
\hline \multirow[t]{3}{*}{ Maternal country of birth } & Nordic countries & 30112 & $(3.5)$ & 860890 & 1.00 & Refe \\
\hline & Europe/USA/ Canada/Australia/NZ & 698 & $(3.4)$ & 20409 & 0.98 & 0.97 \\
\hline & Other & 3773 & $(3.5)$ & 107699 & 1.00 & 0.97 \\
\hline \multirow[t]{3}{*}{ Delivery mode } & Non-instrumental & 23946 & $(2.6)$ & 905290 & 1.00 & Refe \\
\hline & $\mathrm{VE}$ & 10314 & $(12.6)$ & 81932 & 4.76 & 4.66 \\
\hline & Forceps & 323 & $(18.2)$ & 1776 & 6.88 & $6.23-7$ \\
\hline
\end{tabular}

Table 2. Maternal risk factors for OASIS after multivariable modified Poisson regression analyses.

\begin{tabular}{|c|c|c|c|}
\hline & & Adjusted (maternal characteristics (MC)) & Adjusted \\
\hline & & $\mathrm{RR}$ & $(95 \% \mathrm{CI})$ \\
\hline \multirow{3}{*}{ Year of delivery } & $2005-2008$ & 1.00 & Reference \\
\hline & 2009-2012 & 0.94 & $0.92-0.97$ \\
\hline & $2013-2016$ & 0.92 & $0.90-0.95$ \\
\hline \multirow[t]{6}{*}{ Maternal age (years) } & $<20$ & 0.45 & $0.40-0.50$ \\
\hline & $20-24$ & 0.67 & $0.65-0.70$ \\
\hline & $24-29$ & 1.0 & Reference \\
\hline & $30-34$ & 1.24 & $1.21-1.27$ \\
\hline & $34-39$ & 1.29 & $1.25-1.34$ \\
\hline & {$[?] 40$} & 1.23 & $1.15-1.32$ \\
\hline \multirow[t]{3}{*}{ Parity } & 1 & 3.13 & $3.05-3.21$ \\
\hline & 2 & 1.0 & Reference \\
\hline & [?]3 & 0.27 & $0.25-0.29$ \\
\hline \multirow[t]{3}{*}{ Smoking } & Yes & 0.64 & $0.60-0.68$ \\
\hline & No & 1.0 & Reference \\
\hline & Not known & 0.95 & $0.88-1.03$ \\
\hline \multirow[t]{5}{*}{ Body Mass Index $\left(\mathrm{kg} / \mathrm{m}^{2}\right)$} & $<18.5$ & 1.12 & $1.05-1.20$ \\
\hline & $20-24.9$ & 1.0 & Reference \\
\hline & $25-29.9$ & 1.07 & $1.04-1.10$ \\
\hline & [?]30 & 1.04 & $1.04-1.10$ \\
\hline & Not known & 1.01 & $0.95-1.08$ \\
\hline \multirow[t]{5}{*}{ Maternal height $(\mathrm{cm})$} & $<155$ & 1.34 & $1.26-1.42$ \\
\hline & $155-164$ & 1.11 & $1.08-1.13$ \\
\hline & $165-174$ & 1.0 & Reference \\
\hline & {$[?] 175$} & 0.92 & $0.88-0.95$ \\
\hline & Not known & 1.03 & $0.93-1.14$ \\
\hline \multirow[t]{3}{*}{ Maternal education level } & [?]Primary/lower secondary & 0.85 & $0.81-0.89$ \\
\hline & Upper secondary & 0.88 & $0.85-0.90$ \\
\hline & $<3$ yrs higher education & 0.92 & $0.89-0.95$ \\
\hline
\end{tabular}




\begin{tabular}{lllr}
\hline & & Adjusted (maternal characteristics (MC)) & Adjusted \\
\hline & {$[?] 3$ yrs higher education } & 1.00 & Reference \\
& Not known & 0.94 & $0.90-0.98$ \\
Maternal country of birth & 1.00 & Reference \\
& Nordic countries & 0.91 & $0.84-0.98$ \\
& Europe/USA/Canada/Australia/NZ & $1.17-1.25$ \\
Delivery mode & Other & & \\
& Non-instrumental & & \\
& VE & & \\
& Forceps & & \\
\hline
\end{tabular}

Table 3. Birth weight in relation to risk of sphincter rupture

\begin{tabular}{lllllll}
\hline & Sphincter rupture & Sphincter rupture & All vaginal births & Crude & Crude & Adjusted (maternal ch \\
\hline Birth weight $(\mathrm{g})$ & $\mathrm{n}$ & $(\%)$ & $\mathrm{N}$ & $\mathrm{OR}$ & $(95 \% \mathrm{CI})$ & OR \\
$<2500$ & 231 & $(0.9)$ & 25472 & 0.24 & $0.21-0.27$ & 0.17 \\
$2500-2999$ & 1845 & $(1.8)$ & 101202 & 0.48 & $0.45-0.50$ & 0.36 \\
$3000-3499$ & 8641 & $(2.7)$ & 323256 & 0.70 & $0.68-0.72$ & 0.59 \\
$3500-3999$ & 13713 & $(3.8)$ & 357012 & 1.00 & ref & 1.00 \\
$4000-4499$ & 7829 & $(5.2)$ & 149762 & 1.36 & $1.33-1.40$ & 1.65 \\
4500 & 2324 & $(7.2)$ & 32284 & 1.87 & $1.80-1.96$ & 2.84 \\
\hline
\end{tabular}

